\title{
Identification of Beutler-Fano formula in eigenphase shifts and eigentime delays near a resonance
}

\author{
Chun-Woo Lee \\ Department of Chemistry, Ajou University, 5 Wonchun Dong, Suwon 442-749, KOREA
}

\begin{abstract}
Eigenphase shifts and eigentime delays near a resonance for a system of one discrete state and two continua are shown to be functionals of the Beutler-Fano formula using appropriate dimensionless energy units and line profile indices. Parameters responsible for the avoided crossing of eigenphase shifts and eigentime delays are identified. Similarly, parameters responsible for the eigentime delays due to a frame change are identified. With the help of new parameters, analogy with the spin model is pursued for the $S$ matrix and time delay matrix $Q$. The $S$ matrix is found to be put into $\exp [i(a+b \sigma \cdot \hat{n})]$. The time delay matrix $Q$ is shown to be given as $Q=\frac{1}{2} \tau_{r}\left(1+\overrightarrow{P_{a}} \cdot \vec{\sigma}+\overrightarrow{P_{f}} \cdot \vec{\sigma}\right)$ where the first term is the time delay due to resonance, the second term is the one due to avoided crossing interaction and the last term is the one due to a frame change. It is found that $P_{a}^{2}+P_{f}^{2}$ equals unity.

03.65.Ge, 03.80.+r, 33.80Gj, 34.10.+x
\end{abstract}

\section{INTRODUCTION}

Resonances observed in the energy-dependence of atomic and molecular processes often correspond to an autoionization or predissociation observed in photo-absorption, i.e., to the excitation of a quasi-bound state in a continuum region [1:2]. Their spectra are characterized by rich profiles providing a wealth of information on the atomic and molecular dissociation or ionization processes. Beutler's early observation of rare-gas levels auto-ionizing into a single continuum with angular-momentum conservation was reproduced by Fano through the formula [3]:

$$
\sigma(\epsilon)=\sigma_{0} \frac{(\epsilon-q)^{2}}{1+\epsilon^{2}}
$$

where $\epsilon$ is the reduced energy defined by $2\left(E-E_{0}\right) / \Gamma ; E_{0}$ and $\hbar / \Gamma$ represent the resonance energy and the mean life time of the quasi-bound state, respectively; $\sigma_{0}$ represents the photo-fragmentation cross section to the continuum state; $q$ is an index that characterizes the line profile (here $-q$ is employed instead of the usual $+q$ ). Extension of Beutler-Fano formula to the dissociation into multi-channels was obtained by Combet-Farnoux [4]. The energy dependence of $S$ matrix near an isolated resonance for such predissociation or autoionization into multi-channels was obtained long ago and well known [5]. By diagonalizing $S$ matrix, eigenphase shifts $\delta_{i}(i=1,2, \ldots n)$ are obtained from its eigenvalues as

$$
S=U e^{2 i \delta} \tilde{U}
$$

and utilized frequently as a tool for analyzing the resonances [6]-7]. Eigenphase shifts and corresponding eigenchannels are also extensively used in various forms in Multichannel Quantum Defect Theory which is regarded as one of the most general and powerful theories of resonance [1].

In contrast to photo-fragmentation cross sections and $S$ matrix, the analytical formulas for eigenphase shifts in multichannel processes are not known. According to the numerical studies, eigenphase shifts in the multichannel system are known as showing complicated behaviors near a resonance due to the avoided crossing between curves of eigenphase shifts along the energy [6, 8 . Such a phenomenon of the avoided crossing along the energy is not conspicuous in case of the formulas for photo-dissociation cross sections and $S$ matrices. We report here the detailed studies on the behaviors of eigenphase shifts and also times delayed by a collision [9 [10] for the system of one discrete state and two continua, which turn out to be described by functionals of the Beutler-Fano formula. Their behaviors in the system of one discrete state and one continuum are already studied [3]. If one more continuum is added to that system, there enter new phenomena of avoided crossing between curves of eigenphase shifts and eigentimes delayed and of times delayed due to a frame change along the energy besides the resonance behavior. The avoided crossing interactions between eigenphase shifts along the energy always take place near a resonance if they are not excluded for symmetry reasons, as we will see in the later section. The addition of more continua may simply introduce more avoided crossings among the newly added eigenphase shifts. In this sense, the current system of one discrete state and two continua may serve as a prototype system for the study of the effects of avoid crossings between eigenphase shifts and times delayed due to the frame change on the resonance phenomena. 
The import of the current work may be in the findings of the new parameters for the avoided crossing interaction, where $S$ matrix can be put into a form of $e^{i H}$ with $H$ hermitian and the time delay matrix (or life time matrix as called by Smith $[10$ ) can be put into a form of $1+\vec{P} \cdot \vec{\sigma}$ where $\vec{P}$ is a polarization vector and $\vec{\sigma}$ is the Pauli matrix. The analogy with the spin system under a magnetic field is exploited to find further properties of $S$ matrix and time delay matrix.

Section II describes the known properties of eigenphase shifts. Section III describes the form of $S$ matrix near a resonance and the equation for eigenphase shifts. It also discusses some special cases and general characteristics of eigenphase shifts and eigentime delays. Sec. [IV obtains the formulas of eigenphase shifts as functionals of the Beutler-Fano formula for the system of one discrete state and two continua. Sec. V obtains the formulas of eigentime delays as functionals of Beutler-Fano formula.

\section{KNOWN PROPERTIES OF EIGENPHASE SHIFTS}

It is well known that, for an isolated resonance in single channel scattering, the energy dependence of a phase shift, $\delta(E)$, is described by the following formula [1]

$$
\tan \left[\delta(E)-\delta^{0}\right]=\frac{\Gamma}{2\left(E_{0}-E\right)}=-\frac{1}{\epsilon},
$$

where $\delta^{0}, E_{0}$ and $\Gamma$ denote the background phase shift, the energy and the width of the resonance, respectively. The second equality in Eq. (3) defines the dimensionless energy. It can be deduced from Eq. (3) that $\delta(E)$ increases slowly except for the narrow energy range around $E_{0}$ where its value undergoes a rapid change and is eventually increased by $\pi$ as energy varies from $-\infty$ to $\infty$. Due to the property of tangent function, $\delta(E)$ can be determined only up to $n \pi$ ( $n$ integer). It is usually taken to be $\delta^{0}$ at $E \rightarrow-\infty$. Then, it becomes $\delta^{0}+\pi$ at $E \rightarrow \infty$. In the absence of resonance, $\delta$ is equal to $\delta^{0}$. The background phase shift $\delta^{0}$ is a slowly varying function of energy and usually taken to be constant as a good approximation. Thus, the increase of $\delta(E)$ around $E_{0}$ solely comes from resonance. Let us denote the difference between $\delta(E)$ and $\delta^{0}$ by $\delta_{r}(E)$. With this notation, Eq. (3) becomes $\tan \delta_{r}(E)=\tan \bar{\delta}_{r}(\epsilon)=$ $-1 / \epsilon$, and with the mentioned phase convention of setting $\delta(E)$ to $\delta^{0}$ at $E=-\infty$, it can be transformed as follows

$$
e^{i \bar{\delta}_{r}(\epsilon)}=\frac{-\epsilon+i}{\sqrt{\epsilon^{2}+1}}
$$

Macek [8] generalized Eq. (3) to multichannel processes as follows

$$
2\left(E-E_{0}\right)=\sum_{k=1}^{n} \Gamma_{k} \cot \left[\delta_{k}^{0}-\delta_{m}(E)\right], \quad m=1, \ldots, n,
$$

where $\delta_{m}(E)$ is the eigenphase shift defined in Eq. (2), $\delta_{k}^{0}$ is the background phase shifts in channel $k$, and $\Gamma_{k}$ is the partial decay width to channel $k$.

Hazi [1] also showed that the eigenphase sum, which will be denoted as $\delta_{\Sigma}(E)$, satisfies the "single-channel" formula

$$
\delta_{\Sigma}(E) \equiv \sum_{k=1}^{n} \delta_{k}(E)=\delta_{\Sigma}^{0}+\tan ^{-1} \frac{\Gamma}{2\left(E_{0}-E\right)}=\delta_{\Sigma}^{0}-\tan ^{-1} \frac{1}{\epsilon}
$$

where $\Gamma$ is the total width of the resonance, i.e. $\Gamma=\sum_{m=1}^{n} \Gamma_{m}$, and $\delta_{\Sigma}^{0}$ is the sum of the background eigenphase, i.e. $\delta_{\Sigma}^{0}=\sum_{m=1}^{n} \delta_{m}^{0}$. The reduced energy $\epsilon$ is the one defined in Eq. (3) except that $\Gamma$ now denotes the total width.

\section{EQUATIONS FOR EIGENPHASE SHIFTS IN THE NEIGHBORHOOD OF RESONANCE}

The form of $S$ matrix in the neighborhood of an isolated resonance in multichannel processes is well known and repeatedly derived in the past using various resonance theories [2,5]. Though its form is basically same for all resonance theories, its detailed expressions may look differently from theory to theory. Here, Fano's configuration interaction theory is adopted for the treatment of resonance [3]. In the configuration interaction theory, an isolated resonance in multichannel system is treated as regarding the system as composed of one discrete state $\phi$ and many continuum wave functions $\psi_{E}^{-(j)}$. The latter are taken to pre-diagonalize $H$. In other words, it is assumed that continuum 
wave-functions are unable to interact directly with each other. They can, however, interact with each other indirectly via their interactions with the discrete state $\phi$ if the following integrals

$$
V_{j E}=\left(\psi_{E}^{-(j)}|H| \phi\right)
$$

are not zero. This means that $H$ is no longer diagonalized in the combined space spanned by the basis set $\left\{\phi, \psi_{E}^{-(j)}\right\}$. Eigenfunctions $\Psi_{E}^{-(j)}$ of $H$ in the space spanned by $\left\{\phi, \psi_{E}^{-(j)}\right\}$ can be obtained analytically as shown by Fano [3, 4 . By applying the incoming wave boundary conditions to those eigenfunctions as follows

$$
\Psi_{E}^{-(j)} \rightarrow \sum_{j^{\prime}} \phi_{j^{\prime}}(\omega) \sqrt{\frac{m}{2 \pi k_{j^{\prime}}}}\left(e^{i k_{j^{\prime}} R} \delta_{j^{\prime} j}-e^{-i k_{j^{\prime}} R} S_{j^{\prime} j}\right)
$$

S matrix may be obtained 洒 as

$$
S_{j^{\prime} j}=\sum_{j^{\prime \prime}} S_{j^{\prime} j^{\prime \prime}}^{0}\left(\delta_{j^{\prime \prime} j}+2 \pi i \frac{V_{j^{\prime \prime} E} V_{j E}^{\star}}{E-E_{0}-i \pi \sum_{k}\left|V_{k E}\right|^{2}}\right),
$$

where $S_{j^{\prime} j^{\prime \prime}}^{0}$ is the $S$ matrix of the background scattering. Eq. (9) is different from that of outgoing wave in that $i$ is replaced by $-i$. The incoming wave instead of outgoing one is employed here as our interests are in the photodissociation processes.

As stated in Ref. [3], $2 \pi \sum_{k}\left|V_{k E}\right|^{2}$ is the spectral width of the resonance peak and is denoted as $\Gamma$. According to Ref. [5] 8], Eq. (9) may be rewritten as

$$
S_{j^{\prime} j}=\sum_{j^{\prime \prime} j^{\prime \prime \prime}} U_{j^{\prime} j^{\prime \prime}}^{0}\left(e^{-2 i \delta_{j^{\prime \prime}}^{0}} \delta_{j^{\prime \prime} j^{\prime \prime \prime}}+i c_{j^{\prime \prime}} c_{j^{\prime \prime \prime}}\right) \widetilde{U_{j^{\prime \prime \prime} j}^{0}} .
$$

In matrix notation, the above equation becomes

$$
S=U^{0}\left(e^{-2 i \delta^{0}}+i c \tilde{c}\right) \widetilde{U}^{0} \equiv U^{0} A \widetilde{U^{0}},
$$

where $c$ is the column vector and its $k$-th element is defined by

$$
c_{k} \equiv e^{-i \delta_{k}^{0}} \sqrt{\frac{\Gamma_{k}}{E-E_{0}-i \Gamma / 2}} .
$$

$\Gamma_{k}$ is the partial resonance decay width to the background eigenchannel $k$.

Let us consider obtaining eigenphase shifts $\delta$ by the diagonalization of $S$ matrix

$$
S=U e^{-2 i \delta} \tilde{U} .
$$

If $A=e^{-2 i \delta^{0}}+i c \tilde{c}$ is diagonalized as

$$
\begin{gathered}
A=V e^{-2 i \delta} \tilde{V}, \\
S=U^{0} V e^{-2 i \delta} \tilde{V} \widetilde{U^{0}} .
\end{gathered}
$$

From Eqs. (13) and (15), $U=U^{0} V$.

Let $x$ denote the eigenvalue $e^{-2 i \delta}$ of $A$, then the eigenvalue of $A=e^{-2 i \delta^{0}}+i c \tilde{c}$ can be obtained by solving the following secular equation

$$
\left|\begin{array}{cccc}
e^{-2 i \delta_{1}^{0}}+i c_{1}^{2}-x & i c_{1} c_{2} & i c_{1} c_{3} & \cdots \\
i c_{2} c_{1} & e^{-2 i \delta_{2}^{0}}+i c_{2}^{2}-x & i c_{2} c_{3} & \ldots \\
i c_{3} c_{1} & i c_{3} c_{2} & e^{-2 i \delta_{3}^{0}}+i c_{3}^{2}-x & \ldots \\
\cdot & \cdot & \cdot & \ldots \\
\cdot & \cdot & \cdot & \ldots
\end{array}\right|=0 .
$$


If we divide the first row of the above determinant by $i c_{1}$, the second row by $i c_{2}$ and the $k$-th row by $i c_{k}$ in general, and if we introduce $x_{k}$ defined by

$$
x_{k} \equiv \frac{e^{-2 i \delta_{k}^{0}}+i c_{k}^{2}-x}{i c_{k}} \quad\left(\text { or } x_{k}-c_{k}=\frac{e^{-2 i \delta_{k}^{0}}-x}{i c_{k}}\right),
$$

the secular equation becomes

$$
\left|\begin{array}{cccc}
x_{1} & c_{2} & c_{3} & \ldots \\
c_{1} & x_{2} & c_{3} & \ldots \\
c_{1} & c_{2} & x_{3} & \ldots \\
\cdot & \cdot & \cdot & \ldots \\
\cdot & \cdot & \cdot & \ldots
\end{array}\right|=0
$$

The above equation for the system of $n$ continua can be transformed into Sardi's form [12]

$$
\prod_{k=1}^{n}\left(x_{k}-c_{k}\right)+\sum_{k=1}^{n}\left[c_{k} \frac{\partial}{\partial x_{k}} \prod_{m=1}^{n}\left(x_{m}-c_{m}\right)\right]=0 .
$$

By dividing Eq. (19) by its first term, one obtains

$$
\sum_{k=1}^{n} \frac{c_{k}}{c_{k}-x_{k}}=1
$$

By substitution of Eqs. (12) and (17), Eq. (20) becomes

$$
i \sum_{k=1}^{n} \frac{\Gamma_{k}}{e^{-2 i\left(\delta-\delta_{k}^{0}\right)}-1}=E-E_{0}-i \frac{\Gamma}{2} .
$$

Equating the real and imaginary parts, Eq. (21) yields two relations: one is Macek's formula and the other one is $\sum_{k} \Gamma_{k}=\Gamma$. This derivation of Macek's formula is simpler than the original one. The Sardi's form is given as a polynomial of order $\mathrm{n}$ and more convenient than Macek's formula in obtaining the solution. In particular, it shows explicitly that the number of roots of Macek's formula is the same as the number of continua, $n$. We will use it in the next section to obtain the solution for the system of one discrete state and two continua. Before doing that, let us comment on the general properties of eigenphase shifts obtainable from the Macek's formula.

From Macek's formula, we could draw two properties of eigenphase shifts. Firstly, its differentiation with respect to energy yields

$$
\frac{d \delta_{m}}{d E}=2\left[\sum_{k=1}^{n} \frac{\Gamma_{k}}{\sin ^{2}\left(\delta_{k}^{0}-\delta_{m}\right)}\right]^{-1}>0, \quad m=1, \ldots, n
$$

The above equation tells us that the first derivatives of eigenphase shifts with respect to energy near a resonance are positive. Secondly, asymptotic values of eigenphase shifts (at $\left.\left|2\left(E-E_{0}\right) / \Gamma\right| \rightarrow \infty\right)$ are given as $\delta_{1}^{0}, \delta_{2}^{0}, \delta_{3}^{0}, \ldots, \delta_{n}^{0}$ up to multiples of $\pi$.

Let us assume that $\delta_{1}^{0}<\delta_{2}^{0}<\cdots<\delta_{n}^{0}$ and that they are all in the range of $[0, \pi]$, for simplicity, which can be always done without loosing generality by the Hazi's formula ([6). Then, the above two properties on eigenphase shifts tell us that eigenphase shifts vary between two asymptotic values of their abscissas given by $\left[\delta_{1}^{0}, \delta_{2}^{0}\right],\left[\delta_{2}^{0}, \delta_{3}^{0}\right], \ldots,\left[\delta_{n-1}^{0}, \delta_{n}^{0}\right]$. If $\delta_{n}^{0}$ becomes larger than $\pi$, it is shifted by $-\pi$ and increases again toward $\delta_{1}^{0}$. Since the sum of eigenphase shifts shows the resonance behavior and increases by $\pi$ around resonance, each eigenphase shift should similarly show up the resonance behavior between two consecutive asymptotes of the abscissas $\left[\delta_{i}^{0}, \delta_{i+1}^{0}\right], \quad(i=1, \ldots, n)$. When the consecutive eigenphase shifts are the same, let us say $\delta_{i}^{0}=\delta_{i+1}^{0}$, then one of two corresponding eigenphase shifts remains constant while the other one shows up the resonance behavior between two asymptotes $\left[\delta_{i}^{0}, \delta_{i+2}^{0}\right]$.

\section{EIGENPHASE SHIFTS FOR THE SYSTEM OF ONE DISCRETE STATE AND TWO CONTINUA}

Let us now consider the system of one discrete state and two continua. For this system, Eq. (19) becomes 


$$
\left[\left(x_{1}-c_{1}\right)\left(x_{2}-c_{2}\right)+c_{1}\left(x_{2}-c_{2}\right)+c_{2}\left(x_{1}-c_{1}\right)\right]=0 .
$$

Substituting Eq. (17) into (23), we obtain

$$
x^{2}-\left(e^{-2 i \delta_{1}^{0}}+i c_{1}^{2}+e^{-2 i \delta_{2}^{0}}+i c_{2}^{2}\right) x+\left(e^{-2 i \delta_{1}^{0}}+i c_{1}^{2}\right)\left(e^{-2 i \delta_{2}^{0}}+i c_{2}^{2}\right)+c_{1}^{2} c_{2}^{2}=0 .
$$

The roots of the quadratic equation (24) are obtained as

$$
\begin{aligned}
x & =e^{-2 i \delta_{ \pm}(E)} \\
& =\frac{e^{-i\left(\delta_{1}^{0}+\delta_{2}^{0}\right)}}{E-E_{0}-i \frac{\Gamma}{2}}\left(\left(E-E_{0}\right) \cos \Delta_{12}^{0}+\frac{\Delta \Gamma}{2} \sin \Delta_{12}^{0} \pm i\left\{\Gamma_{1} \Gamma_{2}+\left[\left(E-E_{0}\right) \sin \Delta_{12}^{0}-\frac{\Delta \Gamma}{2} \cos \Delta_{12}^{0}\right]^{2}\right\}^{1 / 2}\right),
\end{aligned}
$$

where $\Gamma=\Gamma_{1}+\Gamma_{2}, \Delta \Gamma=\Gamma_{1}-\Gamma_{2}$, and $\Delta_{12}^{0}=\delta_{1}^{0}-\delta_{2}^{0}$. If we introduce a dimensionless energy $\epsilon_{r}$ (taken differently from the usual notation $\epsilon$ in order to avoid the confusion with another dimensionless energy $\epsilon_{a}$ which will be defined later) as

$$
\epsilon_{r} \equiv \frac{E-E_{0}}{\Gamma / 2}
$$

the above equation becomes

$$
e^{-2 i \bar{\delta}_{ \pm}\left(\epsilon_{r}\right)}=\frac{e^{-i\left(\delta_{1}^{0}+\delta_{2}^{0}\right)}}{\epsilon_{r}-i}\left\{\epsilon_{r} \cos \Delta_{12}^{0}+\frac{\Delta \Gamma}{\Gamma} \sin \Delta_{12}^{0} \pm i\left[\frac{4 \Gamma_{1} \Gamma_{2}}{\Gamma^{2}}+\left(\epsilon_{r} \sin \Delta_{12}^{0}-\frac{\Delta \Gamma}{\Gamma} \cos \Delta_{12}^{0}\right)^{2}\right]^{1 / 2}\right\},
$$

where $\bar{\delta}$ is used instead of $\delta$ in order to emphasize that the eigenphase shifts are now functions of $\epsilon_{r}$ instead of $E$.

Analysis of the eigenphase shifts in Eq. (27) is done in the next subsection by introducing phase shifts due to the resonance and avoided crossing interaction.

\section{A. Phase shifts due to resonance and avoided crossing interaction}

Eq. (27) can be decomposed into the product of two terms of unit modulus [13]:

$$
\begin{aligned}
& e^{-i \bar{\delta}_{r}\left(\epsilon_{r}\right)}=\frac{-\sqrt{\epsilon_{r}^{2}+1}}{\epsilon_{r}-i} \\
& e^{ \pm i \bar{\delta}_{a}\left(\epsilon_{r}\right)}=\frac{-1}{\sqrt{\epsilon_{r}^{2}+1}}\left\{\epsilon_{r} \cos \Delta_{12}^{0}+\frac{\Delta \Gamma}{\Gamma} \sin \Delta_{12}^{0} \mp i\left[\frac{4 \Gamma_{1} \Gamma_{2}}{\Gamma^{2}}+\left(\epsilon_{r} \sin \Delta_{12}^{0}-\frac{\Delta \Gamma}{\Gamma} \cos \Delta_{12}^{0}\right)^{2}\right]^{1 / 2}\right\},
\end{aligned}
$$

(the notations $\delta_{r}$ and $\delta_{a}$ without the bar are also used elsewhere if it is desired to express them in terms of $E$ instead of $\epsilon_{r}$ ). The phase shift $\bar{\delta}_{r}$ in the first term is the one due to the resonance as already mentioned in Eq. (4)). In single channel scattering, it is the only term besides the background phase shift contributing to the eigenphase shifts near a resonance. In two channel scattering, there enters another phase shift $\bar{\delta}_{a}$ defined by the second term due to the indirect coupling of two continua via quasi-bound state near a resonance.

The functional dependence of $\bar{\delta}_{a}\left(\epsilon_{r}\right)$ on energy may be best seen by considering its cotangent like $\cot \delta_{r}=-\epsilon_{r}$ as in single channel scattering:

$$
\cot \bar{\delta}_{a}\left(\epsilon_{r}\right)=\frac{-\left(\epsilon_{r} \cos \Delta_{12}^{0}+\frac{\Delta \Gamma}{\Gamma} \sin \Delta_{12}^{0}\right)}{\left[\left(\epsilon_{r} \sin \Delta_{12}^{0}-\frac{\Delta \Gamma}{\Gamma} \cos \Delta_{12}^{0}\right)^{2}+\frac{4 \Gamma_{1} \Gamma_{2}}{\Gamma^{2}}\right]^{1 / 2}}
$$

Let us first rewrite Eq. (30) as follows

$$
\cot \bar{\delta}_{a}\left(\epsilon_{r}\right)=-\cot \Delta_{12}^{0} \frac{\frac{\Gamma \sin \Delta_{12}^{0}}{2 \sqrt{\Gamma_{1} \Gamma_{2}}}\left(\epsilon_{r}+\frac{\Delta \Gamma}{\Gamma} \tan \Delta_{12}^{0}\right)}{\left\{\left[\frac{\Gamma \sin \Delta_{12}^{0}}{2 \sqrt{\Gamma_{1} \Gamma_{2}}}\left(\epsilon_{r}-\frac{\Delta \Gamma}{\Gamma} \cot \Delta_{12}^{0}\right)\right]^{2}+1\right\}^{1 / 2}}
$$


The term inside the bracket in the denominator of Eq. (31) can be transformed as follows

$$
\frac{\Gamma \sin \Delta_{12}^{0}}{2 \sqrt{\Gamma_{1} \Gamma_{2}}}\left(\epsilon_{r}-\frac{\Delta \Gamma}{\Gamma} \cot \Delta_{12}^{0}\right)=\frac{\sin \Delta_{12}^{0}}{\sqrt{\Gamma_{1} \Gamma_{2}}}\left(E-E_{0}-\frac{\Delta \Gamma}{2} \cot \Delta_{12}^{0}\right) .
$$

This suggests that we can introduce a new energy unit $\Gamma_{a}$ :

$$
\Gamma_{a} \equiv \frac{2 \sqrt{\Gamma_{1} \Gamma_{2}}}{\sin \Delta_{12}^{0}}=\frac{\sqrt{\Gamma^{2}-\Delta \Gamma^{2}}}{\sin \Delta_{12}^{0}} .
$$

For simplicity, let us also introduce a new parameter $E_{a}$ :

$$
E_{a} \equiv E_{0}+\frac{\Delta \Gamma}{2} \cot \Delta_{12}^{0}
$$

(later it will be shown that $E_{a}$ is the avoided crossing point energy and $\Gamma_{a}$ is the strength of the avoided crossing interaction).

With these parameters, Eq. (32) becomes $2\left(E-E_{a}\right) / \Gamma_{a}$ which may be considered as a new dimensionless energy and will be denoted as $\epsilon_{a}$ :

$$
\epsilon_{a} \equiv \frac{2\left(E-E_{a}\right)}{\Gamma_{a}}
$$

By substituting Eqs. (32) and (35) into (31), we obtain

$$
\cot \tilde{\delta}_{a}\left(\epsilon_{a}\right)=-\cot \Delta_{12}^{0} \cdot \frac{\epsilon_{a}-q_{a}}{\sqrt{\epsilon_{a}^{2}+1}}
$$

where $\tilde{\delta}_{a}\left(\epsilon_{a}\right)$ is used instead of $\bar{\delta}_{a}\left(\epsilon_{r}\right)$ in order to emphasize that it is now a function of $\epsilon_{a}$ and $q_{a}$ is the new parameter defined by

$$
q_{a} \equiv \frac{-\Delta \Gamma}{\sqrt{\Gamma^{2}-\Delta \Gamma^{2}} \cos \Delta_{12}^{0}} .
$$

Eq. (36) is a functional of the Beutler-Fano function defined by

$$
f_{\mathrm{BF}}(\epsilon, q) \equiv \frac{(\epsilon-q)^{2}}{1+\epsilon^{2}}
$$

and can be rewritten as follows

$$
\cot \tilde{\delta_{a}}\left(\epsilon_{a}\right)=\left\{\begin{array}{ll}
\cot \Delta_{12}^{0} \sqrt{f_{\mathrm{BF}}\left(\epsilon_{a}, q_{a}\right)} & \text { when } \epsilon_{a}<q_{a} \\
-\cot \Delta_{12}^{0} \sqrt{f_{\mathrm{BF}}\left(\epsilon_{a}, q_{a}\right)} & \text { when } \epsilon_{a} \geq q_{a}
\end{array},\right.
$$

Eq. (36) or (39) tells us that the phase shift $\delta_{a}$ due to the avoided crossing interaction takes its simplest form when it is parameterized in terms of $\epsilon_{a}$ and $q_{a}$. Since we want $\Gamma_{a}$ to be positive, it will be assumed that sin $\Delta_{12}^{0} \geq 0$, which can always be achieved by the appropriate choice of 1 and 2 for $\delta_{1}^{0}$ and $\delta_{2}^{0}$. With this convention in mind, $\Gamma_{a}$ will be written as $2 \sqrt{\Gamma_{1} \Gamma_{2}} /\left|\sin \Delta_{12}\right|$ hereafter.

With the above phase shifts, eigenphase shifts are obtained as

$$
2 \delta_{ \pm}(E)=\delta_{1}^{0}+\delta_{2}^{0}+\delta_{r}(E) \pm \delta_{a}(E)
$$

(for the choice of the sign, see [13). It is interesting to note that phase shifts of different origins are added up linearly to eigenphase shifts.

Using Eq. (6), the above equation may be expressed in terms of the eigenphase sum $\delta_{\Sigma}(E)$

$$
2 \delta_{ \pm}(E)=\delta_{\Sigma}(E) \pm \delta_{a}(E)
$$

The eigenphase sum $\delta_{\Sigma}(E)$ consists of the background eigenphase sum $\delta_{\Sigma}^{0}$ and the phase shift $\delta_{r}(E)$ due to the resonance. The phase shifts due to the resonance contribute positively from zero to $\pi$ as energy varies around a resonance. The phase shift is positive since particles are attracted and bound temporarily near a resonance. The 
\pm sign in front of $\delta_{a}(E)$ in Eq. (40) tells us that the phase shift due to the avoided crossing interaction in one eigenchannel increases at the expense of the phase shift of the other eigenchannel and vice versa. If it is increased in one eigenchannel due to attraction, it decreases in the other one due to repulsion. They are exactly canceled out and do not contribute to the eigenphase sum. The study of $\delta_{a}$ as a function of $\epsilon_{a}$ shows that $\delta_{a}$ has an extremum of $\cot ^{-1}\left(\sqrt{q_{a}^{2}+1} \cot \Delta_{12}^{0}\right)$ at $\epsilon_{a}=-1 / q_{a}$ (a minimum when $q_{a}>0$, a maximum when $\left.q_{a}<0\right)$. From its definition, the limiting behavior of $\tilde{\delta}_{a}$ at off-resonance is obtained as

$$
\tilde{\delta}_{a}\left(\epsilon_{a}\right) \rightarrow \begin{cases}\Delta_{12}^{0} & \text { when } \epsilon_{a} \rightarrow-\infty \\ \pi-\Delta_{12}^{0} & \text { when } \epsilon_{a} \rightarrow \infty\end{cases}
$$

[note that $0 \leq \Delta_{12}^{0} \leq \pi / 2$ according to the convention mentioned below Eq. (39)]. $\tilde{\delta}_{a}$ increases around resonance by $\pi-2 \Delta_{12}^{0}$. Thus the phase shift due to avoided crossing interaction varies most around resonance when $\Delta_{12}^{0}=0$ and least when $\Delta_{12}^{0}=\pi / 2$. It passes the middle, $\pi / 2$, of two asymptotes of the abscissas in Eq. (42) when $\epsilon_{a}=q_{a}$. Since the avoided crossing point energy $E_{a}$ corresponds to zero of $\epsilon_{a}$ [see the discussion below Eq. (45)], $q_{a}$ tells how the avoided crossing point energy is apart from the middle of two asymptotes of the abscissas. Such an apartness is a measure of the asymmetry of the curves of $\tilde{\delta}_{a}\left(\epsilon_{a}\right)$. Fig. 1 1 shows the variations in the behaviors of $\delta_{a}$ and $\delta_{ \pm}$as $q_{a}$ varies.

So far, we obtained eigenvalues of $S$ matrix for two open channels near a resonance and decomposed them into contributions from the background, resonance, and avoided crossing interactions. Let us now consider obtaining the eigenvectors of $S$ matrix which are usually called eigenchannels. $S$ matrix is diagonalized in two steps. It is first transformed to $A$ by $U^{0}$ which diagonalizes $S^{0}$ matrix, i.e., $S=U^{0} A \widetilde{U^{0}}\left(S^{0}=U^{0} e^{-2 i \delta^{0}} \widetilde{U^{0}}\right)$. $A$ is then diagonalized as in Eq. (14) by $V$ matrix which is composed of two eigenvectors $v_{+}$and $v_{-}$corresponding to $\delta_{+}$and $\delta_{-}$as

$$
V=\left(v_{+} v_{-}\right) \text {. }
$$

After a lengthy derivation, eigenvectors are obtained as

$$
v_{+}=\left[\begin{array}{c}
\cos \left(\frac{\theta_{a}}{2}\right) \\
\sin \left(\frac{\theta_{a}}{2}\right)
\end{array}\right], \quad v_{-}=\left[\begin{array}{c}
-\sin \left(\frac{\theta_{a}}{2}\right) \\
\cos \left(\frac{\theta_{a}}{2}\right)
\end{array}\right]
$$

with $\theta_{a}$ defined by

$$
\cos \theta_{a} \equiv-\frac{\epsilon_{a}}{\sqrt{1+\epsilon_{a}^{2}}}, \quad \sin \theta_{a} \equiv \frac{1}{\sqrt{1+\epsilon_{a}^{2}}} .
$$

It is interesting to note that eigenvectors are independent of $q_{a}$. They depend only on $\epsilon_{a}$. As $\epsilon_{a}$ varies from $-\infty$ through zero to $\infty, \theta_{a}$ varies from zero through $\pi / 2$ to $\pi$ and $v_{+}$varies from $\left(\begin{array}{l}1 \\ 0\end{array}\right)$ through $\frac{1}{\sqrt{2}}\left(\begin{array}{l}1 \\ 1\end{array}\right)$ to $\left(\begin{array}{l}0 \\ 1\end{array}\right)$. Thus, at $\epsilon_{a}=0$ or at $E=E_{0}+\frac{\Delta \Gamma}{2} \cot \Delta_{12}^{0}$, two eigenphase shifts are avoided most. For this reason $\epsilon_{a}=0$ is considered as the avoided crossing point energy. (Another way to see that $\epsilon_{a}=0$ is the avoided crossing point energy might be to remove the cause of the avoidance of two eigenphase shift curves and to let them cross through each other and to see whether the crossing point is $\epsilon_{a}=0$. This was done and $\epsilon_{a}=0$ is confirmed to be the crossing point.)

Let us parameterize $U^{0}$ matrix as

$$
U^{0}=\left(\begin{array}{cc}
\cos \theta^{0} & -\sin \theta^{0} \\
\sin \theta^{0} & \cos \theta^{0}
\end{array}\right)
$$

where $\theta^{0}$ may be considered as the angle by which the frame for background eigenchannels is rotated from the frame for background asymptotic channels. By calculating the multiplication of $U=U^{0} V$ and by noting that $U$ matrix is composed of eigenvectors $u_{+}$and $u_{-}$of $S$ matrix as

$$
U=\left(u_{+} u_{-}\right)
$$

the eigenvectors of $S$ matrix are obtained as

$$
u_{+}=\left[\begin{array}{c}
\cos \left(\frac{\theta_{a}^{\prime}}{2}\right) \\
\sin \left(\frac{\theta_{a}^{\prime}}{2}\right)
\end{array}\right], \quad u_{-}=\left[\begin{array}{c}
-\sin \left(\frac{\theta_{a}^{\prime}}{2}\right) \\
\cos \left(\frac{\theta_{a}^{\prime}}{2}\right)
\end{array}\right],
$$

where $\theta_{a}^{\prime}$ is defined by

$$
\theta_{a}^{\prime} \equiv \theta_{a}+2 \theta^{0}
$$




\section{B. $S$ matrix in terms of new parameters}

Let us now express $S$ matrix in terms of new parameters introduced in the previous section. Reassembling by using its eigenvalues $e^{-2 i \delta_{+}}$and $e^{-2 i \delta_{-}}\left(\delta_{ \pm}=\delta_{\Sigma} \pm \delta_{a}\right)$ and eigenvectors Eq. (44), $A$ becomes as follows

$$
\begin{aligned}
A & =\left(\begin{array}{ll}
v_{+} & v_{-}
\end{array}\right)\left(\begin{array}{cc}
e^{-2 i \delta_{+}} & 0 \\
0 & e^{-2 i \delta_{-}}
\end{array}\right)\left(\begin{array}{c}
\widetilde{v_{+}} \\
\widetilde{v_{-}}
\end{array}\right) \\
& =e^{-i \delta_{\Sigma}}\left(\begin{array}{cc}
\cos \delta_{a}-i \sin \delta_{a} \cos \theta_{a} & -i \sin \delta_{a} \sin \theta_{a} \\
-i \sin \delta_{a} \sin \theta_{a} & \cos \delta_{a}+i \sin \delta_{a} \cos \theta_{a}
\end{array}\right) .
\end{aligned}
$$

From the relation $S=U^{0} A \tilde{U}^{0}$ with $U^{0}$ matrix defined in (46), $S$ matrix is obtained as

$$
S=e^{-i \delta_{\Sigma}}\left(\begin{array}{cc}
\cos \delta_{a}-i \sin \delta_{a} \cos \theta_{a}^{\prime} & -i \sin \delta_{a} \sin \theta_{a}^{\prime} \\
-i \sin \delta_{a} \sin \theta_{a}^{\prime} & \cos \delta_{a}+i \sin \delta_{a} \cos \theta_{a}^{\prime}
\end{array}\right),
$$

where $\theta_{a}^{\prime}$ is defined in Eq. (49). Using Pauli matrices, it can be transformed into an invariant form:

$$
\begin{aligned}
S & =e^{-i \delta_{\Sigma}}\left\{\cos \delta_{a}-i \sin \delta_{a}\left[\sigma_{z} \cos \theta_{a}^{\prime}+\sigma_{x} \sin \theta_{a}^{\prime}\right]\right\} \\
& =e^{-i \delta_{\Sigma}}\left(\cos \delta_{a}-i \sin \delta_{a} \vec{\sigma} \cdot \hat{n}_{\theta_{a}^{\prime}}\right),
\end{aligned}
$$

where the unit vector $\hat{n}_{\theta_{a}^{\prime}}$ is defined as

$$
\hat{n}_{\theta_{a}^{\prime}} \equiv \hat{z} \cos \theta_{a}^{\prime}+\hat{x} \sin \theta_{a}^{\prime}
$$

Since

$$
\cos \delta_{a}-i \sin \delta_{a} \vec{\sigma} \cdot \hat{n}_{\theta_{a}^{\prime}}=e^{-i \delta_{a} \vec{\sigma} \cdot \hat{n}_{\theta_{a}^{\prime}}},
$$

it is simplified into the form of $e^{i H}$ ( $H$ hermitian):

$$
S=e^{-i\left(\delta_{\Sigma}+\delta_{a} \vec{\sigma} \cdot \hat{n}_{\theta_{a}^{\prime}}\right)}
$$

Since $\left(\vec{\sigma} \cdot \hat{n}_{\theta_{a}^{\prime}}\right)^{2}=1, \vec{\sigma} \cdot \hat{n}_{\theta_{a}^{\prime}}$ has two eigenvalues $\pm 1 . u_{+}$and $u_{-}$in Eq. (48) are their corresponding eigenvectors. In this case, it is well known [14 that the vectors $\pm \hat{n}_{\theta_{a}^{\prime}}$ equal the expectation values of the Pauli spin operator, $\left(u_{ \pm}|\vec{\sigma}| u_{ \pm}\right)$, and are called the polarization vectors that correspond to the eigenstates $u_{ \pm}$, respectively. We can obtain the change in the direction of the polarization axis as a function of energy by differentiating Eq. (54) with respect to $\epsilon_{a}$ :

$$
\frac{d \hat{n}_{\theta_{a}^{\prime}}}{d \epsilon_{a}}=\hat{y} \times \hat{n}_{\theta_{a}^{\prime}} \frac{d \theta_{a}^{\prime}}{d \epsilon_{a}}
$$

Since $\tan \theta_{a}=-1 / \epsilon_{a}$

$$
\frac{d \theta_{a}^{\prime}}{d \epsilon_{a}}=\frac{d \theta_{a}}{d \epsilon_{a}}=\frac{1}{1+\epsilon_{a}^{2}}
$$

The above equations also appear in the spin model for the adiabatic analysis of collisions [15].

\section{TIMES DELAYED BY COLLISION NEAR A RESONANCE}

\section{A. Time delay matrix and eigentime delays}

For a single channel system, times delayed by collision, $\tau$, are obtained by the first derivatives of phase shifts:

$$
\tau(E)=2 \hbar \frac{d \delta(E)}{d E} .
$$

For a multichannel system, we may consider the time delay matrix $Q$ defined by 9,10 


$$
Q=i \hbar\left(S^{\dagger} \frac{d S}{d E}\right)
$$

The unitarity of $S$ matrix ensures that the time delay matrix $Q$ is hermitian. Let us now consider the time delay matrix for the system of one discrete state and two continua. Using Eq. (53) for $S$ matrix expressed in terms of the Pauli matrices, the first derivative of $S$ matrix with respect to energy is calculated as

$$
\frac{d S}{d E}=-i \frac{d \delta_{\Sigma}}{d E} S+e^{-i \delta_{\Sigma}}\left(-\sin \delta_{a}-i \cos \delta_{a} \vec{\sigma} \cdot \hat{n}_{\theta_{a}^{\prime}}\right) \frac{d \delta_{a}}{d E}+e^{-i \delta_{\Sigma}}\left(-i \sin \delta_{a} \vec{\sigma} \cdot \frac{d \hat{n}_{\theta_{a}^{\prime}}}{d E}\right) .
$$

Now the time delay matrix $Q$ is obtained as

$$
i \hbar S^{\dagger} \frac{d S}{d E}=\hbar \frac{d \delta_{\Sigma}}{d E}+\vec{\sigma} \cdot \hat{n}_{\theta_{a}^{\prime}} \hbar \frac{d \delta_{a}}{d E}+\sin \delta_{a} \vec{\sigma} \cdot\left[\hat{y} \times \hat{n}_{\theta_{a}^{\prime}} \cos \delta_{a}-\hat{y} \sin \delta_{a}\right]\left(\hbar \frac{d \theta_{a}^{\prime}}{d E}\right) .
$$

Since $\hat{n}_{\theta_{a}^{\prime}} \times \hat{y}$ and $\hat{y}$ are perpendicular to $\hat{n}_{\theta_{a}^{\prime}}$, their linear combination is also perpendicular to $\hat{n}_{\theta_{a}^{\prime}}$. Let us introduce a vector

$$
\hat{n}_{\theta_{a}^{\prime}}^{\perp} \equiv \hat{y} \times \hat{n}_{\theta_{a}^{\prime}} \cos \delta_{a}-\hat{y} \sin \delta_{a}
$$

perpendicular to $\hat{n}_{\theta_{a}^{\prime}}$ and making an angle $\delta_{a}$ with $\hat{n}_{\theta_{a}^{\prime}} \times \hat{y}$ and also define the following quantities

$$
\begin{aligned}
\tau_{r} & \equiv 2 \hbar \frac{d \delta_{r}}{d E}, \\
\tau_{a} & \equiv 2 \hbar \frac{d \delta_{a}}{d E}, \\
\tau_{f} & \equiv 2 \hbar \sin \delta_{a} \frac{d \theta_{a}^{\prime}}{d E} .
\end{aligned}
$$

The last three quantities correspond to the time delay due to the resonance, the avoided crossing interaction, and the change of the direction of the eigenvectors $\hat{n}_{\theta_{a}^{\prime}}$ of $S$ matrix, or usually termed as the frame change, as a function of energy, respectively. Then, the time delay matrix $Q$ may be written as

$$
Q=\frac{1}{2}\left(\tau_{r}+\vec{\sigma} \cdot \hat{n}_{\theta_{a}^{\prime}} \tau_{a}+\vec{\sigma} \cdot \hat{n}_{\theta_{a}^{\prime}}^{\perp} \tau_{f}\right)
$$

Since observable properties of a particle with spin $\frac{1}{2}$ can be represented as a function of its spin polarization $\vec{P}$ and the states of all the two-level (two-continua here) systems can be mapped on the states of orientation of a particle with spin $\frac{1}{2}, Q$ may be rewritten in terms of the polarization vectors as

$$
Q=\frac{1}{2} \tau_{r}\left(1+\overrightarrow{P_{a}} \cdot \vec{\sigma}+\overrightarrow{P_{f}} \cdot \vec{\sigma}\right)
$$

where polarization vectors are defined as

$$
\overrightarrow{P_{a}} \equiv \frac{\tau_{a}}{\tau_{r}} \hat{n}_{\theta_{a}^{\prime}}, \quad \overrightarrow{P_{f}} \equiv \frac{\tau_{f}}{\tau_{r}} \hat{n}_{\theta_{a}^{\prime}}^{\perp}
$$

The degrees of polarization $\left|\overrightarrow{P_{a}}\right|$ and $\left|\overrightarrow{P_{f}}\right|$ will be denoted simply as $P_{a}$ and $P_{f}$. They are positive by definition (the term polarization may not be adequate for the time delay matrix. We will return to this point later). Let us introduce a new unit vector

$$
\hat{n}_{t}=\hat{n}_{\theta_{a}^{\prime}} \frac{\tau_{a}}{\sqrt{\tau_{a}^{2}+\tau_{f}^{2}}}+\hat{n}_{\theta_{a}^{\prime}}^{\perp} \frac{\tau_{f}}{\sqrt{\tau_{a}^{2}+\tau_{f}^{2}}}
$$

Then Eqs. (65) and (67) become

$$
Q=\frac{1}{2}\left(\tau_{r}+\vec{\sigma} \cdot \hat{n}_{t} \sqrt{\tau_{a}^{2}+\tau_{f}^{2}}\right)=\frac{1}{2} \tau_{r}\left(1+\vec{P}_{t} \cdot \vec{\sigma}\right),
$$

where 


$$
\vec{P}_{t} \equiv \sqrt{P_{a}^{2}+P_{f}^{2}} \hat{n}_{t}
$$

Now eigenvalues of the time delay matrix $Q$ or eigentime delays, which will be denoted as $\tau_{ \pm}$, are easily obtained as

$$
\tau_{ \pm}=\frac{1}{2}\left(\tau_{r} \pm \sqrt{\tau_{a}^{2}+\tau_{f}^{2}}\right)=\frac{1}{2} \tau_{r}\left(1 \pm P_{t}\right) .
$$

Notice that $\tau_{a}$ and $\tau_{f}$ are added up incoherently to eigentime delays. Also because of $\operatorname{tr}\left(\sigma_{i}\right)=0(i=\mathrm{x}, \mathrm{y}, \mathrm{z})$,

$$
\operatorname{tr} Q=\tau_{+}+\tau_{-}=\tau_{r},
$$

that is, the eigentime delay sum has the same form as that of the eigentime delay in the single channel scattering [16].

\section{B. Explicit formulas of time delays}

The time delay due to the resonance, the first term of Eq. (71), is obtained by differentiating $\tan \overline{\delta_{r}}=-1 / \epsilon_{r}$ with respect to $\epsilon_{r}$ as

$$
\bar{\tau}_{r}\left(\epsilon_{r}\right) \equiv 2 \hbar \frac{d \bar{\delta}_{r}\left(\epsilon_{r}\right)}{d \epsilon_{r}}=\frac{2 \hbar}{1+\epsilon_{r}^{2}} .
$$

It takes the Lorentzian form and is thus always positive or delayed as it is kept temporarily by the quasi-bound state.

The form of the time delay due to avoidance, the second term of Eq. (71), is rather complex:

$$
\tau_{a}(E)=2 \hbar \frac{d \delta_{a}}{d E}=\frac{-2 \hbar\left[\frac{1}{2} \Delta \Gamma\left(E-E_{0}\right) \sin \Delta_{12}^{0}-\left(\frac{\Gamma}{2}\right)^{2} \cos \Delta_{12}^{0}\right]}{\left[\left(E-E_{0}\right)^{2}+\left(\frac{\Gamma}{2}\right)^{2}\right]\left\{\Gamma_{1} \Gamma_{2}+\left[\left(E-E_{0}\right) \sin \Delta_{12}^{0}-\frac{1}{2} \Delta \Gamma \cos \Delta_{12}^{0}\right]^{2}\right\}^{1 / 2}} .
$$

By changing the independent variable from $E$ to $\epsilon_{r}$, considering the reduced time delays $\bar{\tau}_{a}\left(=2 \hbar \frac{d \delta_{a}}{d \epsilon_{r}}\right)$, and introducing the new parameters $r^{2}$ and $q_{\tau}$ defined by

$$
\begin{gathered}
r^{2} \equiv \frac{\Gamma^{2}-\Delta \Gamma^{2}}{\Delta \Gamma^{2}}, \\
q_{\tau} \equiv \frac{\Gamma}{\Delta \Gamma} \cot \Delta_{12}^{0},
\end{gathered}
$$

Eq. (74) is simplified as

$$
\bar{\tau}_{a}\left(\epsilon_{r}\right)=-\bar{\tau}_{r}\left(\epsilon_{r}\right) \frac{\epsilon_{r}-q_{\tau}}{\sqrt{\left(\epsilon_{r}-q_{\tau}\right)^{2}+r^{2}\left(1+\epsilon_{r}^{2}\right)}} .
$$

The factor multiplying $\overline{\tau_{r}}\left(\epsilon_{r}\right)$ in the above equation can be transformed to the functional of the Beutler-Fano formula [the Lorentzian shape of $\overline{\tau_{r}}\left(\epsilon_{r}\right)$ may be regarded as a Beutler-Fano shape]

$$
\begin{aligned}
\bar{\tau}_{a}\left(\epsilon_{r}\right) & = \begin{cases}\bar{\tau}_{r}\left(\epsilon_{r}\right) \frac{1}{\sqrt{1+r^{2} \frac{1+\epsilon_{r}^{2}}{\left(\epsilon_{r}-q_{\tau}\right)^{2}}},}, & \text { when } \epsilon_{r} \leq q_{\tau}, \\
-\bar{\tau}_{r}\left(\epsilon_{r}\right) \frac{1}{\sqrt{1+r^{2} \frac{1+\epsilon_{r}^{2}}{\left(\epsilon_{r}-q_{\tau}\right)^{2}}}}, & \text { when } \epsilon_{r}>q_{\tau},\end{cases} \\
& = \begin{cases}\bar{\tau}_{r}\left(\epsilon_{r}\right) \sqrt{\frac{f_{\mathrm{BF}}\left(\epsilon_{r}, q_{\tau}\right)}{f_{\mathrm{BB}\left(\epsilon_{r}, q_{\tau}+r^{2}\right.}},}, & \text { when } \epsilon_{r} \leq q_{\tau}, \\
-\bar{\tau}_{r}\left(\epsilon_{r}\right) \sqrt{\frac{f_{\mathrm{BF}}\left(\epsilon_{r}, q_{\tau}\right)}{f_{\mathrm{BF}}\left(\epsilon_{r}, q_{\tau}\right)+r^{2}}}, & \text { when } \epsilon_{r}>q_{\tau},\end{cases} \\
& \equiv \bar{\tau}_{r}\left(\epsilon_{r}\right) g_{a}\left(\epsilon_{r}\right) .
\end{aligned}
$$

Interestingly, $\bar{\tau}_{a}$ takes the Beutler-Fano formula in the energy scale of $\epsilon_{r}$ instead of $\epsilon_{a}$ in contrast to the case of $\tilde{\delta}_{a}$, though the former is obtained as the derivative of the latter. Notice that the absolute value of $g_{a}$ is the polarization $P_{a}$ considered in the previous section: 


$$
P_{a}=\sqrt{\frac{f_{\mathrm{BF}}\left(\epsilon_{r}, q_{\tau}\right)}{f_{\mathrm{BF}}\left(\epsilon_{r}, q_{\tau}\right)+r^{2}}} .
$$

$g_{a}$ changes its sign at $\epsilon_{r}=q_{\tau}$, so does the polarization vector $\vec{P}_{a}$ at the same energy. The study on $P_{a}$ as a function of $\epsilon_{r}$ shows that $P_{a}$ has a maximum of $\sqrt{\left(1+q_{\tau}^{2}\right) /\left(1+q_{\tau}^{2}+r^{2}\right)}$ at $\epsilon_{r}=-1 / q_{\tau}$ and a minimum of 0 at $\epsilon_{r}=q_{\tau}$ (which corresponds to $\epsilon_{a}=-1 / q_{a}$ where $\delta_{a}$ has an extremum). Fig. 2 shows the variations in the behaviors of $g_{a}\left(\epsilon_{r}\right)$ and $\bar{\tau}_{a}\left(\epsilon_{r}\right)$ as functions of $\epsilon_{r}$ at three different profile indices $q_{\tau}$ with $\Delta_{12}^{0}$ fixed (the value of $q_{\tau}$ is restricted by $\left.\left|q_{\tau} / \cot \Delta_{12}^{0}\right| \geq 1\right)$.

Recalling that $f_{\mathrm{BF}}\left(\epsilon_{r}, q_{\tau}\right) \geq 0$, Eq. (79) tells us that the magnitude of $P_{a}\left(\epsilon_{r}\right)$ is smaller than or equal to unity and has the effect of making the absolute magnitude of the time delay $\bar{\tau}_{a}$ due to an avoidance smaller than or equal to that of the time delay $\bar{\tau}_{r}$ due to a resonance:

$$
\bar{\tau}_{r}\left(\epsilon_{r}\right) \geq\left|\bar{\tau}_{a}\left(\epsilon_{r}\right)\right|
$$

which ensures that $\bar{\tau}_{r}\left(\epsilon_{r}\right) \pm \bar{\tau}_{a}$ are positive.

The above inequality may be obtained without knowing the explicit functional dependences of $\tau_{r}$ and $\tau_{a}$ as functions of energy. Notice that

$$
\tau_{r} \pm \tau_{a}=2 \hbar \frac{d\left(\delta_{r} \pm \delta_{a}\right)}{d E}=2 \hbar \frac{d \delta_{ \pm}}{d E}
$$

Because of the inequality (22), the above equation should be larger than or equal to zero, which proves (80). This inequality is the manifestation of the physical fact that eigenphase shifts are increasing functions of energy in the neighborhood of a resonance as colliding particles are kept bound temporarily around a resonance (attractive forces make positive phase shifts).

The above inequality restricts the magnitude of $P_{a}$ to $0 \leq P_{a} \leq 1$. The magnitude of $P_{a}$ causes the difference between two eigentimes delayed. Eq. (79) tells us that the degree of difference in two eigentime delays is governed by $r^{2}$. Let us consider $r^{2}$ as a function of $x=\Gamma_{1} / \Gamma(0 \leq x \leq 1)$. Then since $r^{2}$ is symmetric with respect to $x=\frac{1}{2}$ and its values are zero at $x=0$ and 1 , infinity at $x=\frac{1}{2}$, the magnitudes of $P_{a}$ are 1 at $x=0$ and 0 at $x=\frac{1}{2}$. Since avoided crossing interaction is strongest at $x=\frac{1}{2}$, the difference in two eigentimes delayed due to an avoided crossing interaction completely disappears when the avoided crossing interaction is strongest.

Let us now consider obtaining the explicit formula of the times delayed due to a frame change. By substituting Eq. (58) and

$$
\sin \delta_{a}=\frac{\Gamma_{a}}{\Gamma} \sin \Delta_{12}^{0} \sqrt{\frac{\epsilon_{a}^{2}+1}{\epsilon_{r}^{2}+1}},
$$

into Eq. (64), the time delay $\tau_{f}$ due to the frame change is obtained as

$$
\tau_{f}=\tau_{r} \sin \Delta_{12}^{0} \sqrt{\frac{\epsilon_{r}^{2}+1}{\epsilon_{a}^{2}+1}} \equiv \tau_{r} g_{f} .
$$

The absolute value of $g_{f}$ is the same as $P_{f}$ :

$$
P_{f}=\left|\sin \Delta_{12}^{0}\right| \sqrt{\frac{\epsilon_{r}^{2}+1}{\epsilon_{a}^{2}+1}},
$$

[actually $g_{f}$ equals $P_{f}$ in the convention mentioned below Eq. (33)]. Fig. 3 shows the variations in the behaviors of $P_{f}\left(\epsilon_{r}\right)$ and $\bar{\tau}_{f}\left(\epsilon_{r}\right)$ as functions of energy at three different profile indices $q_{\tau}$ with $\Delta_{12}^{0}$ fixed.

As in the case of $\tau_{a}, P_{f}$ is smaller than or equal to unity. This can be proved by examining the behavior of the graph of $P_{f}$ as a function of $\epsilon_{r}$. $P_{f}$ can be easily transformed as a function of $\epsilon_{r}$ by substituting

$$
\epsilon_{a}=\frac{\Gamma}{\Gamma_{a}}\left(\epsilon_{r}-\frac{\Delta \Gamma}{\Gamma} \cot \Delta_{12}^{0}\right),
$$

for $\epsilon_{a}$ into Eq. (83). It is then differentiated with respect to energy to yield the roots of the first derivative of $\bar{\tau}_{f}\left(\epsilon_{r}\right)$ at $\epsilon_{r}=-1 / q_{\tau}$ and $q_{\tau}$. From this, it is found that $P_{f}$ has the minimum of $\sin \Delta_{12}^{0} \sqrt{1-(\Delta \Gamma / \Gamma)^{2}}$ at $-1 / q_{\tau}$ and the maximum of unity at $q_{\tau}$. Its values become $\sqrt{1-(\Delta \Gamma / \Gamma)^{2}}$ at $\epsilon_{r} \rightarrow \pm \infty$. This proves $0 \leq P_{f} \leq 1$. 
Now let us consider the magnitude of the total polarization vector $P_{t}=\sqrt{P_{a}^{2}+P_{f}^{2}}$. In order to obtain this, let us further transform Eq. (85) using the definition (33) of $\Gamma_{a}$ and $\Delta \Gamma / \Gamma=\cot \Delta_{12}^{0} / q_{\tau}$ as follows

$$
\epsilon_{a}=\frac{\left|\sin \Delta_{12}^{0}\right|}{\sqrt{1-\left(\frac{\cot \Delta_{12}^{0}}{q_{\tau}}\right)^{2}}}\left(\epsilon_{r}-\frac{\cot ^{2} \Delta_{12}^{0}}{q_{\tau}}\right) .
$$

Also after some manipulation, we obtain the following relation:

$$
\left(\epsilon_{r}-q_{\tau}\right)^{2}+r^{2}\left(\epsilon_{r}^{2}+1\right)=\left(\frac{q_{\tau}}{\cot \Delta_{12}^{0}}\right)^{2}\left[\left(\epsilon_{r}-\frac{\cot ^{2} \Delta_{12}^{0}}{q_{\tau}}\right)^{2}+\frac{1-\left(\frac{\cot \Delta_{12}^{0}}{q_{\tau}}\right)^{2}}{\sin ^{2} \Delta_{12}^{0}}\right] .
$$

Using Eq. (86) to calculate $\epsilon_{a}^{2}+1$ and comparing the latter with the right hand side of Eq. (87), we obtain

$$
\epsilon_{a}^{2}+1=\frac{\sin ^{2} \Delta_{12}^{0}}{r^{2}}\left[\left(\epsilon_{r}-q_{\tau}\right)^{2}+r^{2}\left(\epsilon_{r}^{2}+1\right)\right],
$$

where $r^{2}=\left(q_{\tau} / \cot \Delta_{12}^{0}\right)^{2}-1$ is used. Substituting Eq. (88) into Eq. (84), $P_{f}$ can be put into a functional of Beutler-Fano formula:

$$
P_{f}=\frac{r}{\sqrt{f_{\mathrm{BF}}\left(\epsilon_{r}, q_{\tau}\right)+r^{2}}}
$$

From Eqs. (89) and (79), we obtain the surprising result:

$$
P_{a}^{2}+P_{f}^{2}=1
$$

This means that eigentime delays for the system of one discrete state and two continua are zero and $\tau_{r}\left(\epsilon_{r}\right)$. Though time delays due to the avoided crossing interaction and frame change are asymmetric with respect to the resonance energy and therefore the energy of the longest lifetime does not match with the resonance energy, the energy of the longest overall eigentimes delayed is exactly matched with the resonance energy.

\section{RESULTS AND DISCUSSION}

Let us summarize the results. In this paper, we considered eigenphase shifts and eigentime delays near a resonance for a system of one discrete state and two continua using Fano's configuration interaction theory. The eigenphase shifts are obtained as

$$
2 \delta_{ \pm}(E)=\sum_{i} \delta_{i}^{0}+\delta_{r}(E) \pm \delta_{a}(E),
$$

where $\delta_{r}(E)$ is the phase shift due to the modification of the scattering wave by the quasi-bound state and given by $-\arctan \left(1 / \epsilon_{r}\right)$ and $\delta_{a}(E)$ is the one due to the modification of the scattering wave by the other wave through the indirect interaction via the quasi-bound state and given as a functional of the Beutler-Fano formula:

$$
\cot \delta_{a}(E)=-\cot \Delta_{12}^{0} \frac{\epsilon_{a}-q_{a}}{\left(1+\epsilon_{a}^{2}\right)^{1 / 2}} .
$$

In the above formula, energy is expressed in the unit of $\Gamma_{a}=2 \sqrt{\Gamma_{1} \Gamma_{2}} /\left|\sin \Delta_{12}^{0}\right|$ and shifted from the resonance energy $E_{0}$ by $\frac{\Delta \Gamma}{2} \cot \Delta_{12}^{0}$. The shape of the curve of $\delta_{a}(E)$ is characterized by the line profile index $q_{a}=-2 \Delta \Gamma /\left(2 \sqrt{\Gamma_{1} \Gamma_{2}} \cos \Delta_{12}^{0}\right)$. The strength of the avoided crossing interaction felt by the eigenphase shifts is governed by the magnitude of $\Gamma_{a}$. No shift is expected when $\Gamma_{1}$ equals $\Gamma_{2}$, where the strength of the avoided crossing interaction is strongest. The maximum shift obtains when either $\Gamma_{1}$ or $\Gamma_{2}$ is zero.

Eigenvectors $v_{ \pm}$of $A$ of $S=S^{0} A$ corresponding to eigenvalues $\delta_{ \pm}(E)$ are obtained as

$$
v_{+}=\left[\begin{array}{c}
\cos \left(\frac{\theta_{a}}{2}\right) \\
\sin \left(\frac{\theta_{a}}{2}\right)
\end{array}\right], \quad v_{-}=\left[\begin{array}{c}
-\sin \left(\frac{\theta_{a}}{2}\right) \\
\cos \left(\frac{\theta_{a}}{2}\right)
\end{array}\right],
$$


where $\theta_{a}$ is defined by

$$
\cos \theta_{a}=-\frac{\epsilon_{a}}{\sqrt{1+\epsilon_{a}^{2}}}, \quad \sin \theta_{a}=\frac{1}{\sqrt{1+\epsilon_{a}^{2}}} .
$$

Eigenvectors are independent of $q_{a}$. They depend only on $\epsilon_{a}$. Since $q_{a}$ stands for the apartness of the avoided crossing point energy from the middle of asymptotes of the abscissas of two eigenphase shifts, the above result means that the characteristics of avoided crossing interactions are independent of the asymptotes of the abscissas of eigenphase shifts. The corresponding eigenvectors of $S$ matrix are obtained by replacing $\theta_{a}$ with $\theta_{a}^{\prime}=\theta_{a}+2 \theta_{0}$.

With the new parameters, $S$ matrix is found to be expressible as

$$
S=e^{-i\left(\delta_{\Sigma}+\delta_{a} \vec{\sigma} \cdot \hat{n}_{\theta_{a}^{\prime}}\right)},
$$

where $\hat{n}_{\theta_{a}^{\prime}}=\hat{z} \cos \theta_{a}^{\prime}+\hat{x} \sin \theta_{a}^{\prime}$. In terms of Pauli matrices, the time delay matrix $Q=i \hbar S^{\dagger} \frac{d S}{d E}$ is found to be consisted of three terms:

$$
Q=\frac{1}{2}\left(\tau_{r}+\vec{\sigma} \cdot \hat{n}_{\theta_{a}^{\prime}} \tau_{a}+\vec{\sigma} \cdot \hat{n}_{\theta_{a}^{\prime}}^{\perp} \tau_{f}\right),
$$

the one due to the resonance, the one due to the avoided crossing interaction, and the one due to the change of frame as a function of energy. Because of the last term, eigenfunctions of $Q$ matrix are different from those of $S$ matrix.

The time delay due to the resonance takes a symmetric Lorentzian form and the time delay due the to avoided crossing takes a form of a functional of the Beutler-Fano formula:

$$
\tau_{a}(E)= \begin{cases}-\tau_{r}(E) \frac{1}{\sqrt{1+r^{2} \frac{1+\epsilon_{r}^{2}}{\left(\epsilon_{r}-q_{\tau}\right)^{2}}}}, & \text { when } \epsilon_{r} \leq q_{\tau}, \\ \tau_{r}(E) \frac{1}{\sqrt{1+r^{2} \frac{1+\epsilon_{r}^{2}}{\left(\epsilon_{r}-q_{\tau}\right)^{2}}}}, & \text { when } \epsilon_{r}>q_{\tau} .\end{cases}
$$

The asymmetry of $\tau_{a}$ as a function of energy is brought about by the nonzero value of $q_{\tau}$ which is proportional to the shift of the avoided crossing point energy from the resonance one. Thus the asymmetry of $\tau_{a}$ is caused by the mismatch in the positions of the avoided crossing point and resonance energies.

The time delay due to frame change takes the following form:

$$
\tau_{f}(E)=\tau_{r}(E) \sin \Delta_{12}^{0} \sqrt{\frac{\epsilon_{r}^{2}+1}{\epsilon_{a}^{2}+1}} .
$$

The above form may be understood from the fact that the changes of the frame spanned by the eigenvectors of $S$ matrix is governed by $1+\epsilon_{a}^{2}$ while the time delay due to the resonance is governed by $1+\epsilon_{r}^{2}$. Therefore the ratio of $\tau_{a}$ to $\tau_{r}$ will be a function of $\left(1+\epsilon_{r}^{2}\right) /\left(1+\epsilon_{a}^{2}\right) \cdot \tau_{f}(E)$ is also found to be transformed into a functional of the Beutler-Fano formula.

In analogy with the spin $\frac{1}{2}$ system, the time delay matrix $Q$ may be expressed in terms of polarization vectors and the Pauli spin matrices as

$$
Q=\frac{1}{2} \tau_{r}\left(1+\vec{P}_{a} \cdot \vec{\sigma}+\vec{P}_{f} \cdot \vec{\sigma}\right)
$$

where polarization vectors are defined by

$$
\overrightarrow{P_{a}}=\frac{\tau_{a}}{\tau_{r}} \hat{n}_{\theta_{a}^{\prime}}, \quad \overrightarrow{P_{f}}=\frac{\tau_{f}}{\tau_{r}} \hat{n}_{\theta_{a}^{\prime}}^{\perp}
$$

Like the spin $\frac{1}{2}$ system, it is found that the absolute values of $\vec{P}_{a}$ and $\vec{P}_{f}$ are restricted to $0 \leq\left|\vec{P}_{a}\right| \leq 1$ and $0 \leq\left|\vec{P}_{f}\right| \leq 1$. In the present case a complete depolarization means that eigentimes delays are the same regardless of eigenchannels, while a complete polarization means that eigentime delays are 0 and $\tau_{r}(E)$ as a function of energy. Eigenvectors for eigentime delays due to an avoided crossing interaction and due to a frame change are orthogonal to each other and contribute to the total eigentime delays as $\sqrt{\tau_{a}^{2}+\tau_{f}^{2}}=\tau_{r} \sqrt{P_{a}^{2}+P_{f}^{2}}$. It is found that $P_{a}^{2}+P_{f}^{2}=1$. This means that one among two total eigentime delays is zero while the other one is the same as the time delayed by the resonance state. Though time delays due to an avoided crossing interaction and a frame change are asymmetric with respect to the 
resonance energy and therefore the energy of the longest lifetime not matched with the resonance energy, the energy of the longest overall eigentimes delayed is exactly matched with the resonance energy. Though this is a surprising result, it should rather be so if we recall that all the partial life times obtained from the partial photo-dissociation cross sections as a function of energy are the same in the system of one discrete state and many continua. But the detailed study on their connection is beyond the scope of this paper.

Present work reveals the dynamical parameters that govern the behaviors of eigenphase shifts and eigentime delays for the system of one discrete state and two continua. It may be applied to the systems of more than two continua by approximating that such systems are a cascade of independent two interacting continua. In that sense, current study may be considered as a prototype model for the "isolated" avoided crossing interaction. For the system of one discrete state and three or four continua, Eq. (19) becomes third and fourth order equation and its solution can be obtained by the Cartan and Ferrari's method. It may be highly desirable to do the similar studies on these systems.

\section{ACKNOWLEDGMENTS}

I would like to thank to Professors Fano and Nakamura for their interests and advice on this work. Careful readings

and comments by Young-Man Han are also appreciated. This work was supported by KOSEF under contract No. 961-0305-050-2 and at the end stage by Korean Ministry of Education through Research Fund No. BSRI-97-3449.

[1] U. Fano and A. R. P. Rau, Atomic Collisions and Spectra (Academic, Orlando, 1986).

[2] J. R. Taylor, Scattering Theory (John Wiley and Sons, New York, 1972).

[3] U. Fano, Phys. Rev. 124, 1866 (1961); U. Fano and J. W. Cooper, Phys. Rev. A 137, 1364 (1965).

[4] C.W. Lee, Bull. Korean Chem. Soc. 16, 850 (1995); F. J. Combet-Farnoux, J. Chem. Phys. 25, 287 (1982).

[5] W. Brenig and R. Haag, Fortschr. Phys. 7, 183 (1959); H. Feshbach, Ann. Phys. (N.Y.) 19, 287 (1962).

[6] P. G. Burke, J. Cooper, and S. Ormonde, Phys. Rev. 183, 245 (1969); C. J. Goebel and K. W. McVoy, Phys. Rev. 164, 1932 (1967).

[7] D.W. Schwenke and D.G. Truhlar, J. Chem. Phys. 87, 1095 (1987); S.W. Cho, A.F. Wagner, B. Gazdy, and J.M. Bowman, J. Chem. Phys. 96, 2812 (1992).

[8] J. Macek, Phys. Rev. A 2, 1101 (1970).

[9] For an introduction to time delays, see Ref. [1].

[10] F.T. Smith, Phys. Rev. 118, 349 (1960); Phys. Rev. 130394 (1963); E. Wigner, Phys. Rev. 98, 145 (1955); L. Eisenbud, dissertation, Princeton, June, 1948 (unpublished); B.A. Lippmann, Phys. Rev. 151, 1023 (1966); J.O. Hirschfelder, Phys. Rev. A 19, 2463 (1979); For the chemical applications of time delay matrix, see H.W. Jang, S.E. Choi, J.C. Light, J. Chem. Phys. 100, 4188 (1994) and other references are therein; For more references, see R.G. Newton, Scattering Theory of Waves and Particles (Springer-Verlag, New York, 1982) p327.

[11] A. U. Hazi, Phys. Rev. A 19, 920 (1979); R.K. Nesbet, Adv. At. Mol. Phys. 13, 315 (1977).

[12] T. Muir, A Treatise on the Theory of Determinants (Dover, New York, 1960), p. 97.

[13] With the choice of the sign for $\delta_{a}$ as in Eq. (29), we obtain the following formulas:

$$
\sin \delta_{a}=\frac{\Gamma_{a}}{\Gamma} \sin \Delta_{12}^{0} \sqrt{\frac{1+\epsilon_{a}^{2}}{1+\epsilon_{r}^{2}}}, \quad \cos \delta_{a}=-\frac{\Gamma_{a}}{\Gamma} \cos \Delta_{12}^{0} \frac{\epsilon_{a}-q_{a}}{\sqrt{1+\epsilon_{r}^{2}}},
$$

whose forms are consistent with those for $\theta_{a}$ and $\delta_{r}$ defined in Eqs. (45) and (4).

[14] L.I. Schiff, Quantum Mechanics (McGraw-Hill, New York, 1955) p381-384; U. Fano, Rev. Mod. Phys. 29, 74 (1957).

[15] U. Fano, Phys. Rev. A 19, 410 (1979).

[16] Z.W. Wang and K.T. Lu, Phys. Rev. A 31, 1515,1521 (1985). 

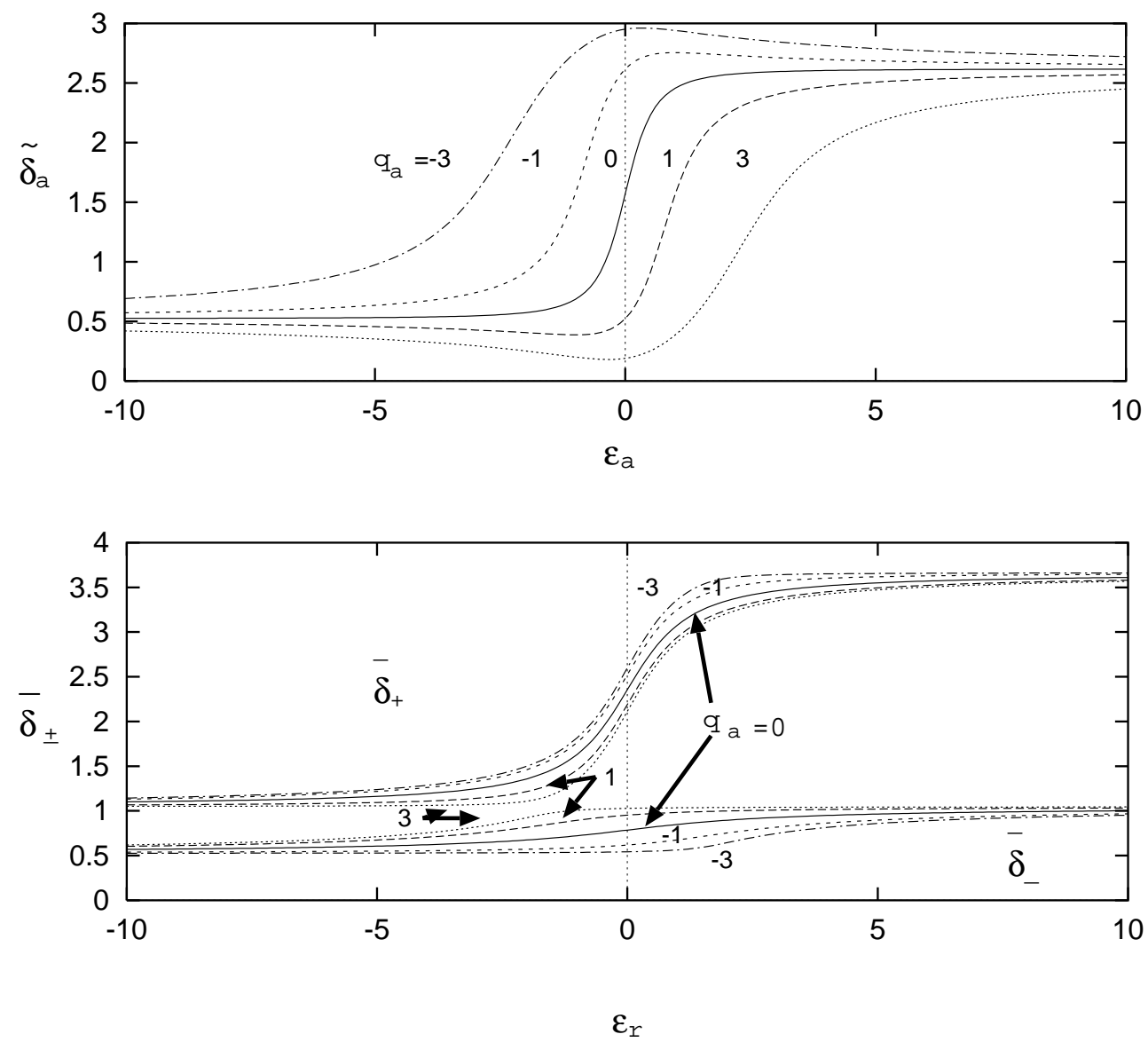

Fig. 1

(az5666, pra)

FIG. 1. $\tilde{\delta}_{a}(E)$ vs. $\epsilon_{a}$ and $\bar{\delta}_{ \pm}\left(\epsilon_{r}\right)$ vs. $\epsilon_{r}$ are plotted for $q_{a}=0, \pm 1$, and \pm 3 . Values for $\delta_{1}^{0}$ and $\delta_{2}^{0}$ are $\pi / 3$ and $\pi / 6$, respectively. 

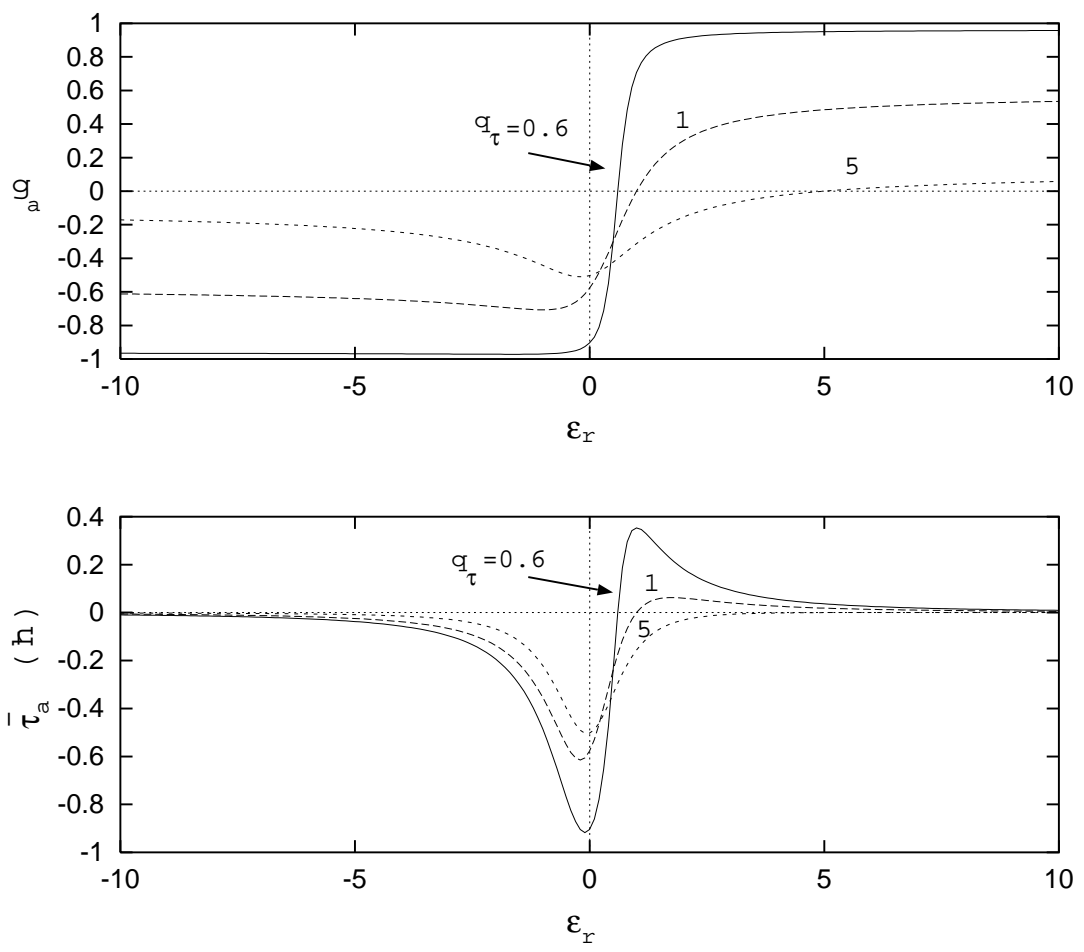

Fig. 2

(az5666, pra)

FIG. 2. $g_{a}\left(\epsilon_{r}\right)$ and $\bar{\tau}_{a}\left(\epsilon_{r}\right)$ vs. $\epsilon_{r}$ are plotted for three different profile indices $q_{\tau}=0.6,1$, and 5 with $\Delta_{12}^{0}=$ $\pi / 3$. 

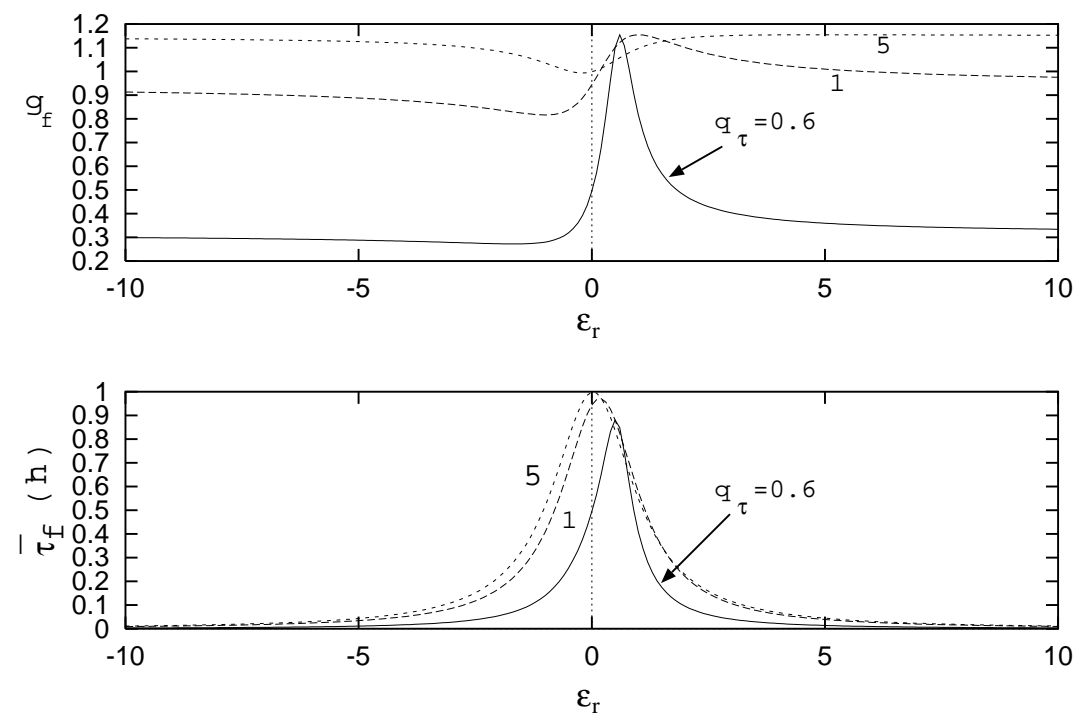

Fig. 3

(az5666, pra)

FIG. 3. $g_{f}\left(\epsilon_{r}\right)$ and $\bar{\tau}_{f}\left(\epsilon_{r}\right)$ vs. $\epsilon_{r}$ are plotted for three different profile indices $q_{\tau}=0.6,1$, and 5 with $\Delta_{12}^{0}=\pi / 6$. 